Journal of Applied Fluid Mechanics, Vol. 3, No. 2, pp. 53-63, 2010.

Available online at www.jafmonline.net, ISSN 1735-3572, EISSN 1735-3645.

DOI: $10.36884 / \mathrm{jafm} .3 .02 .11888$

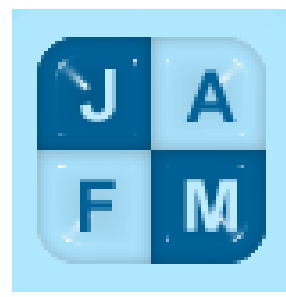

\title{
Computational and Experimental Investigations of Boundary Layer Tripping
}

\author{
M.R. Heidari ${ }^{1}$, M.R. Soltani ${ }^{2}$, M. Farahani ${ }^{3}$ and M. Taeibi-Rahni ${ }^{2}$ \\ ${ }^{1}$ Aerospace Engineering Faculty, Space Research Center, Tehran, Iran. \\ ${ }^{2}$ Sharif University of Technology, Aerospace Engineering Department, Tehran, Iran. \\ ${ }^{3}$ Sharif University of Technology and Engineering Research Institute, Tehran, Iran \\ Email: mrezaheidari@yahoo.com
}

(Received April 14, 2009; accepted July 10, 2009)

\begin{abstract}
Supersonic flow over a tapered body of revolution has been investigated both experimentally and numerically. The experimental study consisted of a series of wind tunnel tests on an ogive-cylinder body. Static pressure distributions on the body surfaces at several longitudinal cross sections, as well as the boundary layer profiles at various angles of attack have been measured. Further, the flow around the model was visualized using Schlieren technique. Tests with a natural development of the boundary layer and with tripping were also carried out. All tests were conducted in the trisonic wind tunnel of Qadr Research Center. Our results show that artificial boundary layer tripping has minor effect on the static surface pressure distribution (depending on its diameter and installation location), while the changes in total pressure around the body were significant. Tripping the boundary layer increased its thickness, changed its profile particularly near the body surface. Two oblique shock waves were formed in the front and behind the trip wire. In this study, using multi-block grid, the thin layer Navier-Stokes (TLNS) equations were solved around the above models. Also patched method was used near the interfaces. Good agreements were achieved when the numerical results were compared with the corresponding experimental data.
\end{abstract}

Key words: Supersonic flow, pressure distribution, boundary layer tripping, long axisymmetric body

\section{NOMENCLATURE}

$\begin{array}{lll}\mathrm{Cp}= & \text { Pressure Coefficient } \\ \mathrm{d}= & \text { Body Diameter } \\ \mathrm{L} / \mathrm{d}= & \text { Fineness Ratio } \\ \mathrm{M}= & \text { Mach Number } \\ \mathrm{Re}_{t r}= & \text { Transition Reynolds Number }\end{array}$

Subscripts

$\infty=$

Free Stream

\section{INTRODUCTION}

Artificial boundary layer tripping by a surface roughness element will generally cause earlier transition, because of additional disturbances it feeds into the boundary layer. Two different geometries exist: two-dimensional roughness (a cylindrical stretch across the flow) and three-dimensional roughness (a sphere or spike or single grain of sand). These are single roughness. There is also the possibility of distributed roughness, such as sandpaper or rows of cylinder or multiple rivets. Two or three-dimensional roughness $\mathrm{x}=$ Longitudinal Position from the Nose

$\mathrm{z}=$ Perpendicular Distance from the Top of Body Surface

$\alpha=$ Angle of Attack (degrees)

$\delta^{*}=$ Displacement Thickness

$\theta=$ Circumferential Angle (Degrees) has quite different effects. The wire wake introduces disturbances to raise the level of Tollmein-Schlichting waves growing downstream. If the wire height is much smaller than the local displacement thickness $\left(\delta^{*}\right)$, there is little effect and transition occurs far away from the wire, (White 1991). Trip strip reduces $\operatorname{Re}_{t r}$ and moves the location of transition toward the wire hence one can control the transition location. There are several investigations on the effects of diameter and place of trip wire on transition location for flow over flat plat, especially in an incompressible flow. The effect of 
roughness on the laminar-turbulent transition in supersonic flow is much less than that in the incompressible flow. The critical roughness height for supersonic flow is about three to seven times greater than that for the incompressible flow. Experiments by Korkegi (1956) at a Mach number of $\mathrm{M}=5.8$ show that even a trip wire may introduce no turbulence at all (Schlichting 1979).

There are many different reasons to study transition. Variations of transition location can affect boundary layer and flow separation. Along the long bodies, the problem of flow separation and boundary layer growth at various flight conditions are very important. Performance of various control surfaces, especially those located close to the end of the body, varies if the flow over them separates. Boundary layer growth and its separation affect the aerodynamic characteristics, particularly drag force and stability criterion, both of which have important roles in the vehicle performance and its mission implementations (Cebeci 1986). Previous parametric boundary layer tripping result for passive trip on a number of blunted conical geometries has served as a basic tool for developing improved semi-empirical analytic methods to predict the tripping effects applicable to general basic bodies and trip configurations (Ericsson and Beyers 1997). The drag measurement and its correlation will be more accurate if the transition point, which is very important in supersonic cruise performance, is known a priori (Goodsel et al. 2000). Boundary layer trips can act as the passive control of compressible boundary layer growth. It may be desirable to promote boundary layer growth from laminar to turbulent state at an early stage of the boundary layer development using some appropriate tripping mechanism (Chou and Childs 1985).

There are few experimental results about trip strip used in three-dimensional bodies, such as a missile configuration. In the present paper, artificial boundary layer tripping, using a single roughness element on axisymmetric bodies is considered both experimentally and numerically. A series of wind tunnel tests on a long axisymmetric body were performed to investigate the pressure distributions, boundary layer profiles, and the flow characteristics at various angles of attack and at a constant supersonic Mach number of 1.6. The effects of cross sectional area variations on the surface static pressure distribution and on the boundary layer profiles were thoroughly investigated. This was done by installing two belts (strips) having different cut-off angles on the cylindrical portion of the model. By changing the belt angles, different bodies were generated and the effects of varying the body cross section on the pressure signatures and on the boundary layer profiles were studied.

In the numerical part of this work, a stationary turbulent supersonic axisymmetric flow over the same body was investigated, using a computer code developed for this research (MBTLNS; Heidari and Taiebi-Rahni 2005). We have assumed adiabatic walls. The flow domain was blocked in streamwise direction and patched method was used in the block boundaries. In each block, the thin layer Navier-Stokes (TLNS) equations were solved using the implicit delta form finite difference with Beam and Warming central differencing scheme (Beam and Warming 1978). For turbulence modeling, the algebraic two layer model of Baldwin and Lomax (1978) was used. The shock waves were captured using shock capturing method. Our computational results for zero angle of attack, Mach number of 1.6, and Reynolds number of $8 \times 10^{6}$ for flow over an axisymmetric ogive-cylinder with two sets of strips with 5 degrees angle were compared with our experimental results.

\section{GoVERNING EQUATIONS AND COMPUTATIONAL METHOdology}

The turbulent supersonic flow over a long axisymmetric body is computationally simulated, in the absence of body forces and heat sources, using multi-block grid. The variations of viscous fluxes in the streamwise direction are assumed negligible.

\subsection{Governing Equations}

The TLNS equations are obtained by neglecting the viscous terms in the streamwise direction. The dimensionless and conservative forms of these equations in curvilinear coordinates $(\xi, \eta)$ are as follows:

$\frac{\partial \mathbf{Q}}{\partial \mathrm{t}}+\frac{\partial \mathbf{F}}{\partial \xi}+\frac{\partial \mathbf{G}}{\partial \eta}+j \mathbf{H}=\frac{1}{\operatorname{Re}}\left[\frac{\partial \mathbf{G}_{\mathrm{v}}}{\partial \eta}+j \mathbf{H}_{\mathrm{v}}\right]$,

where, $j=0$ for two-dimensional and $j=1$ for axisymmetric flows. Also, the dependent variable $\mathbf{Q}$ is: $\mathbf{Q}^{T}=J^{-1}[\rho, \rho u, \rho \mathrm{v}, E]$,

where,

$E=\rho\left(e+\frac{u^{2}+v^{2}}{2}\right)$,

and $\mathrm{J}^{-1}$ is the inverse jacobian matrix (transformation from $\mathrm{x}, \mathrm{y}$ to $\xi, \eta$ coordinates). The viscous/inviscid fluxes are as follows:

$\mathbf{F}=J^{-1}\left(\begin{array}{l}\rho U \\ \rho u U+\xi_{x} p \\ \rho \mathrm{v} U+\xi_{y} p \\ (E+p) U\end{array}\right), \mathbf{G}=J^{-1}\left(\begin{array}{l}\rho V \\ \rho u V+\eta_{x} p \\ \rho \mathrm{v} V+\eta_{y} p \\ (E+p) V\end{array}\right), \quad \mathbf{H}=(y J)^{-1}\left(\begin{array}{l}\rho \mathrm{v} \\ \rho u \mathrm{v} \\ \rho \mathrm{v}^{2} \\ (E+p) \mathrm{v}\end{array}\right)$,

$\mathbf{G}_{\mathrm{v}}=\mu J^{-1}\left(\begin{array}{l}0 \\ b_{1} u_{\eta}+b_{3} \mathrm{v}_{\eta} \\ b_{3} u_{\eta}+b_{2} \mathrm{v}_{\eta} \\ \frac{1}{2} b_{1}\left(u^{2}\right)_{\eta}+\frac{1}{2} b_{2}\left(\mathrm{v}^{2}\right)_{\eta}+b_{3}(u \mathrm{v})_{\eta}+b_{4} \kappa\end{array}\right)$,

$\mathbf{H}_{\mathrm{V}}=\mu(y J)^{-1}\left(\begin{array}{l}0 \\ \eta_{y} u_{\eta}+b_{5} \eta_{x} \\ \eta_{y} \mathrm{v}_{\eta}+b_{5} \eta_{y}-2 \frac{\mathrm{v}}{y} \\ b_{6} \eta_{y}+b_{7} \eta_{x}-\frac{2}{3} \frac{\mathrm{v}^{2}}{y}\end{array}\right)$,

where, 
$U=\xi_{x} u+\xi_{y} \mathrm{v}, \quad V=\eta_{x} u+\eta_{y} \mathrm{v}$,

$b_{1}=\frac{4}{3} \eta_{x}^{2}+\eta_{y}^{2}, \quad b_{2}=\eta_{x}^{2}+\frac{4}{3} \eta_{y}^{2}, \quad b_{3}=\frac{1}{3} \eta_{x} \eta_{y}, \quad b_{4}=\eta_{x}^{2}+\eta_{y}^{2}, \quad \kappa=\gamma(\operatorname{Pr})^{-1} e_{\eta}$,

$b_{5}=\mathrm{v}_{\eta}-\frac{2}{3} y\left(\frac{\mathrm{v}}{y}\right)_{\eta}, b_{6}=\frac{1}{2} u_{\eta}^{2}+\frac{2}{3} \mathrm{v}_{\eta}^{2}-\frac{2}{3} y\left(\frac{\mathrm{v}^{2}}{y}\right)_{\eta}+\kappa$,

$b_{7}=u \mathrm{v}_{\eta}-\frac{2}{3} \mathrm{v} u_{\eta}-\frac{2}{3} y\left(\frac{u \mathrm{v}}{y}\right)_{\eta}$.

\subsection{Turbulence Modeling and Boundary Conditions}

In this work, the governing equations have been Reynolds averaged and the Baldwin-Lomax turbulence model has been used. This model is frequently used because of its simplicity and its reliability. Even though in some flow situations, it is less than $10 \%$ accurate compared to some other models, it can reduce the amount of computations up to about $50 \%$.

The initial pressure, density, and velocity on the surface and along the symmetric axis were obtained. Far field boundary condition was used at infinity and a relatively simple and logical initial guess was shown to be good enough. In the shock capturing technique used, the boundary at infinity was assumed to be a curved bow shock. Assuming two arbitrary values for two empirical coefficients in the equation of the curved bow shock, one can determine how large and up to what points the flow domain extends. At the outflow, all flow variables were determined, using linear interpolation of the interior values. Whereas on the symmetric axis, the governing equations were solved and right under this axis, the mirror symmetric condition was used. This way, quantities such as the velocity perpendicular to the symmetric axis, its derivatives, and the pressure and the temperature gradients were all set to zero.

\subsection{Computational Methodology}

The TLNS equations were discretized using the Beam and Warning scheme as follows:

$$
\begin{aligned}
& \Delta \mathbf{Q}^{n}=\frac{\theta_{1} \Delta t}{1+\theta_{2}} \frac{\partial}{\partial t}\left(\Delta \mathbf{Q}^{n}\right)+\frac{\Delta t}{1+\theta_{2}} \frac{\partial \mathbf{Q}^{n}}{\partial t}+\frac{\theta_{2}}{1+\theta_{2}} \Delta \mathbf{Q}^{n-1} \\
& +O\left[\left(\theta_{1}-\frac{1}{2}-\theta_{2}\right)(\Delta t)^{2}+(\Delta t)^{3}\right],
\end{aligned}
$$

where, $\Delta \mathbf{Q}^{\mathrm{n}}=\mathbf{Q}^{\mathrm{n}+1}-\mathbf{Q}^{\mathrm{n}}$. This scheme is second order implicit with coefficients $\theta_{1}=1$ and $\theta_{2}=0$. In $\xi$ and $\eta$ directions, the above equation becomes:

$$
\begin{aligned}
& \left.\left\{\mathbf{I}+\Delta t\left[\frac{\partial}{\partial \xi}\left(\frac{\partial \mathbf{F}}{\partial Q}\right)^{n}+\frac{\partial}{\partial \eta}\left(\frac{\partial \mathbf{G}}{\partial Q}\right)^{n}+j\left(\frac{\partial \mathbf{H}}{\partial Q}\right)^{n}-\frac{1}{\operatorname{Re}}\left[\frac{\partial}{\partial \eta}\left(\frac{\partial \mathbf{G}_{\mathrm{v}}}{\partial Q}\right)^{n}+j\left(\frac{\partial \mathbf{H}_{\mathrm{v}}}{\partial Q}\right)^{n}\right]\right]\right\}\right\} \Delta \mathbf{Q}^{n}=\mathrm{RHS} \\
& \text { RHS }=-\Delta t\left[\frac{\partial \mathbf{F}^{n}}{\partial \xi}+\frac{\partial \mathbf{G}^{n}}{\partial \eta}+j \mathbf{H}^{n}-\frac{1}{\operatorname{Re}}\left(\frac{\partial \mathbf{G}_{\mathrm{v}}}{\partial \eta}+j \mathbf{H}_{\mathrm{v}}\right)^{n}\right]+\mathrm{D}_{\mathrm{E}}
\end{aligned}
$$

where, I is a $4 \times 4$ unit matrix. Our numerical methodology leads to solving diagonal block matrices in each computational space direction. A simple and fast solution technique is the use of digonalization of the Jacobean matrix of the left hand side (implicit part). Note, the implicit and the explicit artificial viscosity terms $\left(D_{E}\right)$ have been used to smooth out the numerical instabilities.
The first step in grid generation is the correct nodal distribution along the body surface. Then is the blocking of the domain and finally comes the grid generation inside each block. Since we are not ignoring the viscosity, much finer grid is required near the surfaces. Also, finer grid is required in the block, which may contain shock waves, flow separation, or other high flow gradient regions. For most blocks, where there are relatively simple geometries, algebraic grid generation is usually used.

The location of block interfaces is very important. In this work, the blocks were structured in the streamwise direction. For blocking of the domain, one needs to first estimate different flow phenomena and the complexity of the body geometry. Then, the block interfaces are located. Here, we used patched method at the interfaces. Note, whenever connected meshes are used, the lines from one block are continued into the next block. This way, interpolation is not usually required at the interfaces. Besides the limitations this may bring along, it eliminates the errors due to non-conservative data along the interfaces.

In this work, a suitable linear interpolation technique was used for computations at points of a block extended into the neighboring block. Thus, the distribution of lines in the left and the right sides of the interfaces is quite arbitrary and without any limitations. After dividing the flow domain into several blocks, the information set for each block is obtained. This set includes the block numbers, the interface numbers and types, the geometric locations of the corners of each block, and the numbers and types of the neighboring interfaces. Also, other internal information of each block, such as the number of nodes and their arrangements, the CFL number, the artificial viscosity coefficient, etc., have to be known before the flow solution is performed.

\section{EXPERIMENTAL EQUIPMENTS AND TESTS}

All tests were conducted in the trisonic wind tunnel of Qadr Research Center, QRC. The equipments used for this investigation included: Schlieren visualization system, A/D board, traversing mechanism, rake, vacuum pump, manometer, pressure transducer, multiplexer board, Trip wire, computer, and data acquisition software.

\subsection{Tunnel}

The QRC wind tunnel is an open-circuit blow down tunnel and operates continuously between Mach numbers $0.4-2.2$, via engine RPM and nozzle adjustments. It has a test section of $60 \times 60 \times 120 \mathrm{~cm}^{3}$ and is equipped with various internal strain gauge balances for force and moment measurements, pressure transducers, Schlieren visualization system, etc (Soltani et al. 2005)

\subsection{Model, Belts and Trip Strip}

The model used for our studies had a fineness-ratio of 2.5 and a circular-arc, ogival nose tangent to a 
cylindrical after body with $\mathrm{L} / \mathrm{d}=15$ (Fig. 1.a). The model was equipped with 36 static pressure ports located longitudinally and circumferentially. To study the effects of cross section changes, two belts with various inclination angles were installed on the model (Fig. 1b). In this paper, model 1 is used when referring to the simple model (the model without belts), and model 2 for the model with belts $(5,5)$ degrees. A cylindrical wire was installed on model as seen in Figs. 1a and $1 \mathrm{~b}$ This wire has rough surface (to produce more disturbances) and its diameter varies from 1.14, 0.9 , and $0.28 \mathrm{~mm}$ and was installed at $\mathrm{x} / \mathrm{d}=1.75,4.25$, and 6.25 on all two models.

\subsection{Traversing Mechanism}

The traversing system, which was designed and built particularly for this investigation, is capable of moving the rake perpendicular to the body axis with small steps of about $0.003 \mathrm{~mm}$ in $\mathrm{z}$ direction. This system was installed on the $\alpha$-mechanism such that the tubes (pitot total pressure) of the rake were always parallel to the model. The entire mechanism is fully controlled by the computer. Figure 2 shows schematic of the model, traversing system, and the rake. The dashed lines in this figure present model at angle of attack.

\subsection{Tests}

As mentioned before, various tests were conducted to study flow characteristics along the model. The free stream Mach number was 1.6, while the angle of attack was varied between -2 to 6 degrees. At each angle of attack and for all models, total pressure data were obtained by the rake at a longitudinal station of $\mathrm{x} / \mathrm{d}=11.25$ for at least 11 locations in $\mathrm{z}$ direction. An accurate linear potentiometer was used to determine the distances between the body surface and the rake. All experimental data shown in this paper are ensemble average of several hundred data, taken several times to ensure repeatability.

\section{RESUltS AND DiscuSSIONS}

The length of both models used was $60 \mathrm{~cm}$, while its diameter (d) was $4 \mathrm{~cm}$ (all lengths were normalized using this diameter). Not, the grid points near the nose were much more than those towards the end.

Figure 3 shows the blocks, the grids, and the Mach contours for model 1. As shown in this figure, there are 5 blocks having $2.55 \mathrm{~d}, 1.35 \mathrm{~d}, 0.6 \mathrm{~d}, 2.0 \mathrm{~d}$, and $8.5 \mathrm{~d}$ lengths, respectively. The number of grids in the spanwise direction is 40 , while it is 150 in the streamwise direction (for all blocks). As noted from this figure, since the trip strip is located in the third block, the number of grids in this block is about four times more, compared to its neighboring blocks, while it is about 15 times more, compared to the fifth block. Figure 4 shows the blocks, the grids, and the density contours for model 2. Here, there are again 5 blocks having $1.5 \mathrm{~d}, 0.75 \mathrm{~d}, 1.25 \mathrm{~d}, 3.5 \mathrm{~d}$, and $8.0 \mathrm{~d}$ lengths, respectively. The number of grids in the spanwise direction is 40, while it is 90 and 150 for the second and the rest of the blocks in the streamwise direction, respectively. Note that the trip strip is located in the second block, whose number of grids is about twice that of its neighboring blocks and ten times that of the fifth block.

Schlieren visualization technique was used to study the shock shape (and its variations with angle of attack), formed around the model nose and places where the area changes and in front and rear of the trip strip.

Figure 5 shows the nose shock waves as well as the shock wave and the expansion waves on the trip strip, at zero angle of attack. The numerical results are also shown for comparison. The boundary layer thickness at the trip strip location on Fig. $5 b$ is very thin, so the upper surface of the wire is located in supersonic flow and causes shock formation at its front and rear portions. From this figure, it is seen by inspection that there exists relatively close correlation between the numerical and the experimental results for both cases.

Figure 6 shows the flow field over the cylindrical part of model 1 with trip strip installed at $\mathrm{x} / \mathrm{d}=4.25$. In this station, the boundary layer is thicken relative to the flow over the nose (Fig. 1a), but its sublayer thickness is very small and even for thin wire with $0.28 \mathrm{~mm}$ in diameter, the oblique shock is formed ahead and behind the trip strip. The wire acts as a cylinder, so the incoming flow accelerates when passes along it and becomes supersonic, hence an oblique shock will form to reduce its velocity. For two different wires, there is symmetry in the upper and lower surfaces of the model. Geometrical characteristics of shocks are shown in Fig. 7. In this figure the model angle of attack and the boundary layer thickness are shown. The differences between the shocks shape and the boundary layer thickness is seen quantitatively in this figure.

The effect of adding trip strip on experimental and numerical longitudinal pressure distributions are compared for model 1 and model 2 with trip strip at $\alpha=0$. The experimental and the numerical data compares well up to $\mathrm{x} / \mathrm{d}=12$. However, for $\mathrm{L} / \mathrm{d}>12$, the experimental data for this angle of attack differs from both theoretical and CFD predictions. The differences are probably due to the base flow affecting the boundary layer on the model surface near the end of the model. The measurement errors are calculated and are shown in the experimental data (Fig. 8). The effect of trip strip on experimental longitudinal pressure distributions is shown in Fig. 9 at $\alpha=0$ and 6 degrees for model 1. Results show that the trip strip with 0.9 $\mathrm{mm}$ in diameter has more effects on the pressure distribution near its installation place, i.e., $\mathrm{x} / \mathrm{d}=4.25$, while the presence of the trip strip affects slow variations in surface pressure distribution far away from this station.

The effect of boundary layer tripping on the experimental circumferential pressure data at $\mathrm{x} / \mathrm{d}=3,6$ and 11.5 and at zero angle of attack are shown in Fig. 10 for model 1. For the first station, trip strip has no considerable effects, while for the other two stations (located after the wire) the variations are clearly visible. Similar results are presented in Fig. 11 for model 2. 
Again, the trip strip has only considerable effects on the stations located closed to its installation point.

Figure 12 compares the circumferential pressure distribution at $\mathrm{x} / \mathrm{d}=3$ and 6 at an angle of attack 6 degree for model 2 with and without the trip strip. At $\mathrm{x} / \mathrm{d}=11.5$, no considerable effects are observed. Note that, by increasing angle of attack, the pressure taps located at zero circumferential angle, $\theta=0$, will be located at the leeward side of the model. Therefore, their static pressures decrease, as expected. As $\alpha$ increases, the vortices separate from the nose and extend to the end of the body. These vortices are in general asymmetric and cause asymmetry of the circumferential pressure distributions on the leeward side of the body (Moore 2000). In addition, sensitivity of the model installation in the wind tunnel is high due to its large fineness ratio; hence a small error can cause a considerable value for the side slip angle. Note, the experimental flow field study showed that the flow has a small yaw angle (Masdari 2003). The effects of varying model cross section (by addition of a belt with different angles) on the longitudinal and circumferential pressure distribution are completely discussed in (Soltani et al. 2005). Comparison of the boundary layer profiles for model 1 for cases with and without trip strip at $\mathrm{x} / \mathrm{d}=11.25$ is presented in Fig. 13 for $\alpha=0,4^{\circ}$. From this figure, it is clearly seen that the trip strip increases the thickness of the boundary layer and deforms its shape relative to the clean body. However, for the model with trip strip, the velocity profile never reaches its free stream value. As seen from this figure (Fig. 13), at a distance of about $40 \mathrm{~mm}$ above the model, the measured Mach number is still less than that of the free stream (1.6). This decrease in Mach number is due to the oblique shocks located ahead and behind the wire, decreasing the total pressure. Hence, it reduces the local Mach number (Fig. 6).

Similar trends are seen when studying the effects of trip strip on the boundary layer profiles for model 2 at different angles of attack (Fig. 14). For this model, difference between the Mach number outside the boundary layer is much less than model 1 for clean and tripped body, while, even the clean body due to oblique shocks in the front and rear the belt has Mach number less than free stream. Comparison of Figs. 13 and 14 shows that the trip strip on the nose has less effect than the one installed on stations far away from the nose.

Figure 15 shows Mach contours for the model 2 (model with $5^{\circ}$ belt), at an angle of attack of 2 degrees and at $\mathrm{x} / \mathrm{d}=11.25$, with and without the trip strip. These contours are combinations of angles of attack of 2 and 2 degrees. From this figure, it is clearly seen that addition of the trip strip has increased the boundary layer thickness significantly. Further, it is seen that the flow around the body at this station is fairly symmetric.

\section{Conclusions}

An extensive experimental and numerical study on a long axisymmetric tapered body at a supersonic speed was performed to investigate the pressure distributions and boundary layer profiles at various angles of attack.
Schlieren technique was used to visualize the flow and shock formation at varies conditions. Artificial boundary layer tripping, using the single cylindrical wire, has more effect on the boundary layer thickness and shape and has less effect on the surface pressure distribution, especially for distances far away from the trip strip. Furthermore the location of the trip strip installation and the value of its diameter show significant effects on the total and the static pressure distributions. Trip strip increases the thickness of the boundary layer for both models 1 and 2 . Decreasing the diameter of the trip strip (up to $0.28 \mathrm{~mm}$ ) and changing its installation point toward, the end of the body (to $\mathrm{x} / \mathrm{d}=6.25$ ) could not remove the oblique shock formation ahead and behind the wire. The shape of the boundary layer near the wall, as well as its thickness, varied significantly due to the presence of the trip strip. For both models, the value of $\partial M / \partial z$ at $\mathrm{z}=0$ was considerably different, when compared to the one without trip strip. The Mach contours at $2^{\circ}$ angle of attack confirm the above findings.

Further, a supersonic flow is computationally simulated with the same free stream conditions and geometry as our experimental efforts. Structured multi-block grid and Baldwin-Lomax turbulence model was used to solve the TLNS equations. The numerical scheme was implicit central differenced Beam and Warming, while adaptive patching method was used at the block interfaces. Some of the results were compared with the experimental data. The numerical and experimental results compare well. Also, at the block interfaces, there were not any discontinuities, showing suitable accuracy of the numerical results. The flow shock and expansion waves were clearly shown and the velocity was quite continuous across the block interfaces.

\section{REFERENCES}

Baldwin, B.S. and H. Lomax (1978). Thin-layer approximation and algebraic model for separated turbulent flow. AIAA Paper 78-257, the $16^{\text {th }}$ Aerospace Sciences Meeting, Huntsville, Alabama.

Beam, R.M. and R.F. Warming (1978). An implicit factored scheme for the compressible NavierStokes equation. AIAA J. 16(4), 393-402.

Cebeci, T. (1986). Three Dimensional Boundary Layer on Missiles, Tactical Missile Aerodynamics. Progress in Astronautics and Aeronautics, AIAA 104, 723-777.

Chou, J.H. and M.E. Childs (1985). The Passive control of compressible boundary layer growth by boundary layer trips. AIAA-85-0561.

Ericsson, L.E. and M.E. Beyers (1997). Active and passive boundary layer tripping. AIAA-97-729.

Goodsell, A.M., R.A. Kennelly and J.L. Lawrence (2000). Accounting for laminar run and trip drag in supersonic cruise performance testing. AIAA-000265 . 
Heidari, M.R. and M. Taiebi-Rahni (2005, October). Multi-block computational \& experimental investigations of supersonic turbulent flows around complex bodies of revolution. International, Computational Fluid Dynamic Journal 14(3), 279295.

Korkegi, R.H. (1956). Transition studies and skinfriction measurements on an insulated flat plate at a Mach number of 5.8. JAS 23, 97-102.

Masdari, M. (2003). The Flow Study in the Test Section of the Transonic Wind Tunnel of Qadr Research Center. M. Sc. Thesis, Aerospace Engineering Dept., Sharif Univ. of Technology, Tehran, Iran.
Moore, F.G. (2000). Approximation Methods for Weapon Aerodynamics. Progress in Astronautics and Aeronautics, AIAA 186, Reston.

Schlichting, H. (1979). Boundary-Layer Theory. McGraw-Hill.

Soltani, M.R., M. Taiebi-Rahni, M. Farahani and M.R. Heidari (2005). Flow measurements around a long axisymmetric body with varying cross section. AIAA-05-50.

White, F.M. (1991). Viscous Fluid Flow. $2^{\text {nd }}$ Edition, McGraw-Hill, Singapore.

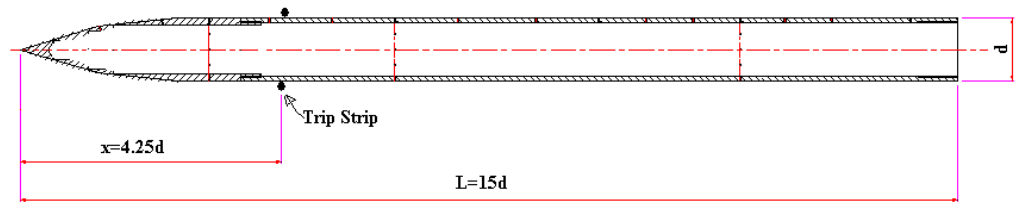

a) Simple model (model 1)

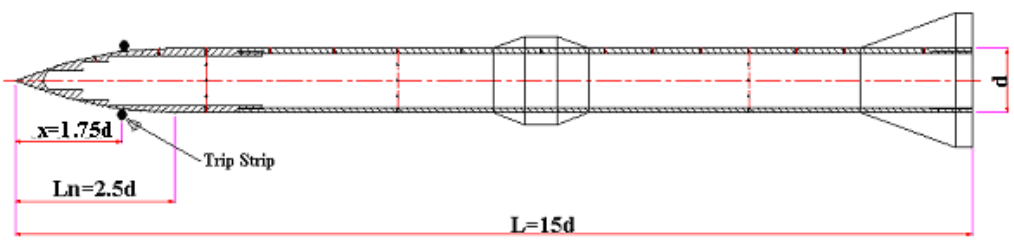

b) Model with belt (model 2)

Fig. 1. Schematic of the transonic wind tunnel and the models.

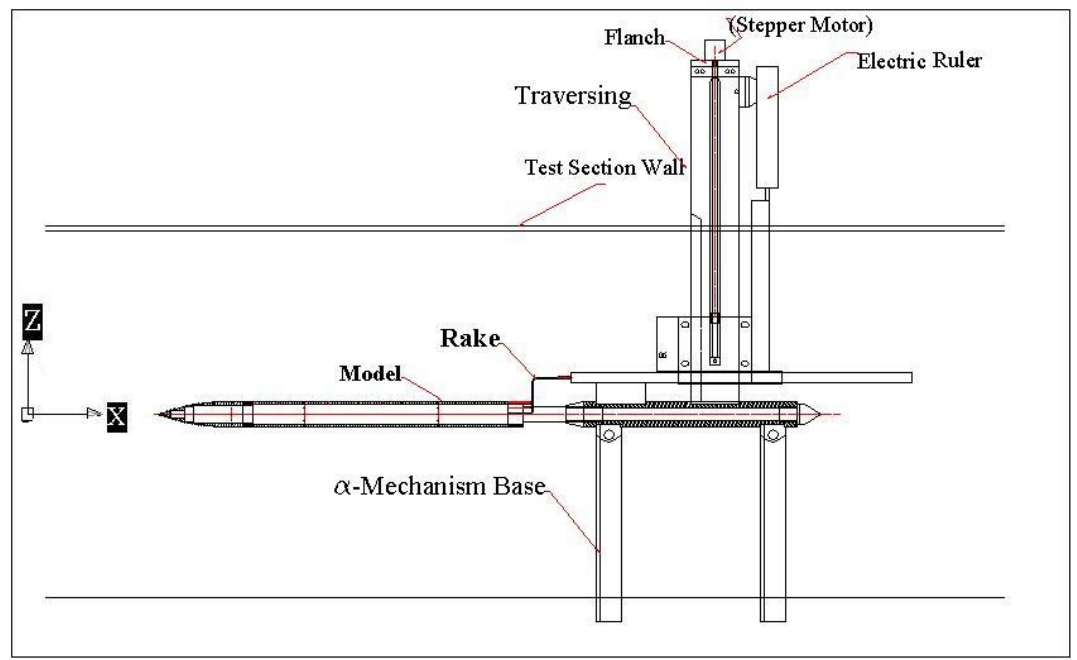

Fig. 2. Schematic of the model inside the wind tunnel with traversing mechanism. 


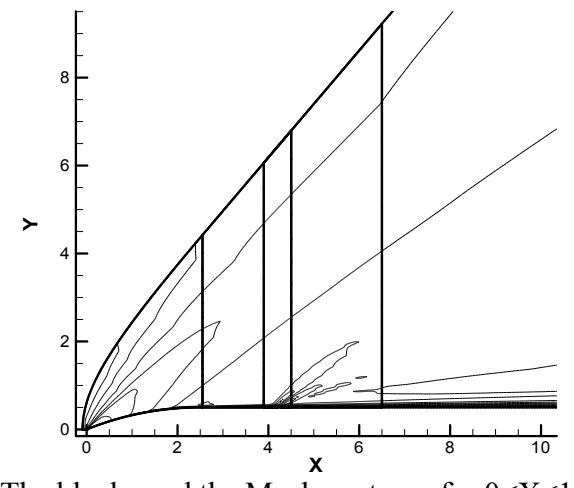

The blocks and the Mach contours for $0<\mathrm{X}<10$

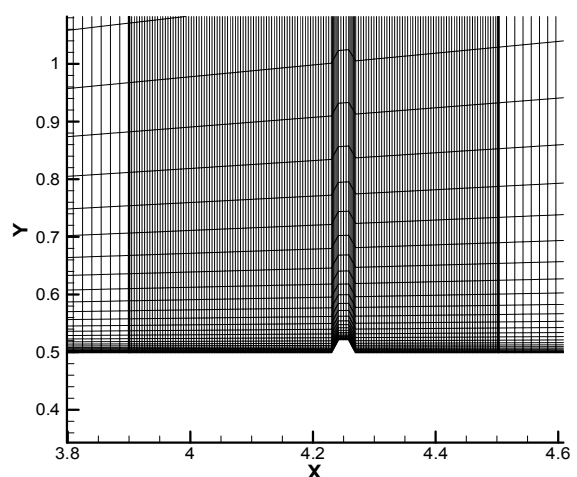

The blocks and the grids for $3.8<\mathrm{X}<4.6$

Fig. 3. The blocks, the related grids used, and the Mach contours for model $1(X=x / d)$.

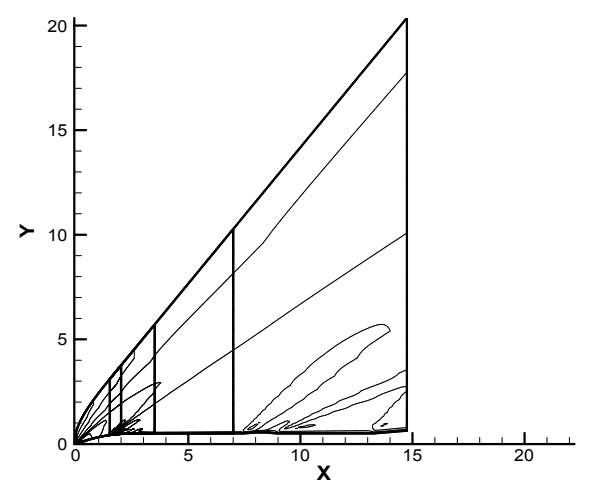

The blocks and the density contours for $0<\mathrm{X}<15$

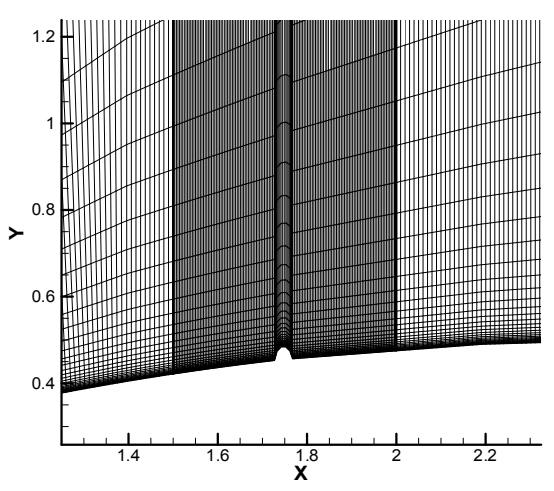

The blocks and the grids for $1.25<\mathrm{X}<2.35$

Fig. 4. The blocks, the related grids used, and the density contours for model 2.

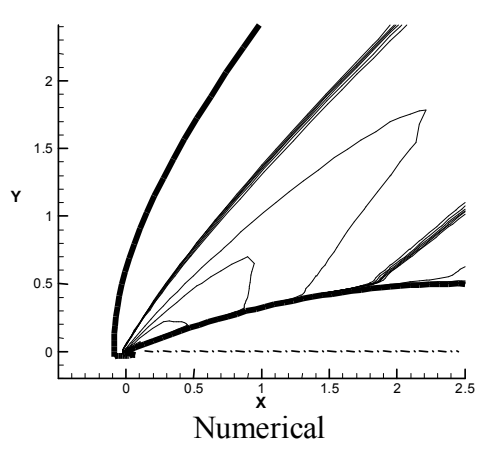

(a) Clean model

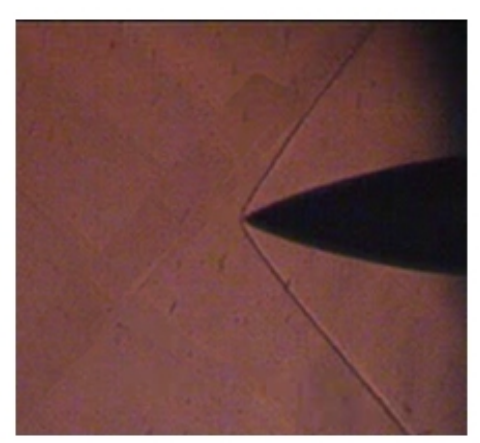

Experimental

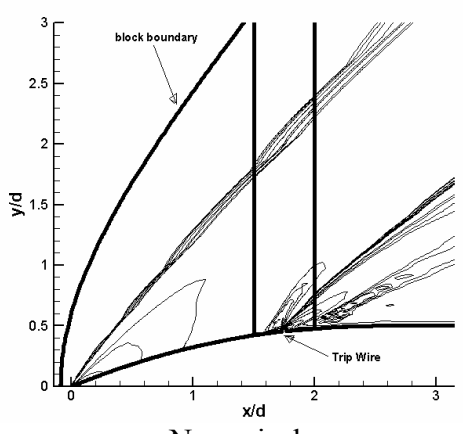

Numerical

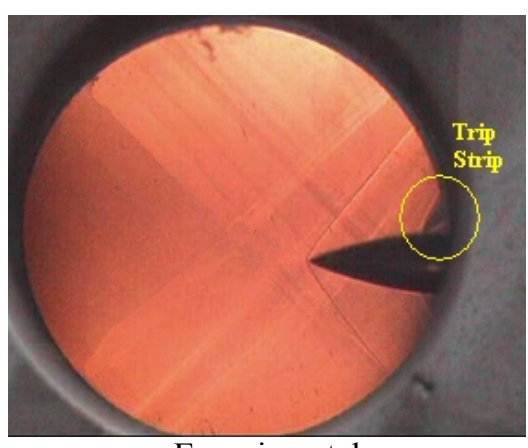

Experimental

(b) Model with $1.14 \mathrm{~mm}$ in diameter trip strip, installed at $\mathrm{x} / \mathrm{d}=1.75$ on the nose

Fig. 5. Experimental (Schlieren photograph) and numerical (pressure contours) flow field over the nose of the model at $\alpha=0$. 

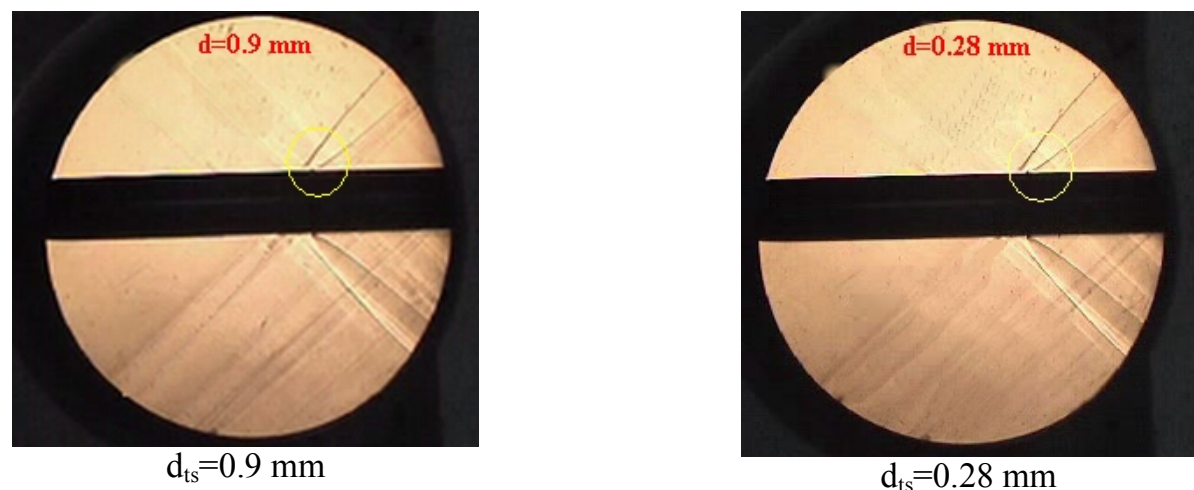

Fig. 6. Flow around cylindrical part of the Model 1 with trip strip installed at $\mathrm{x} / 6=6.25$ and $\alpha=0$.

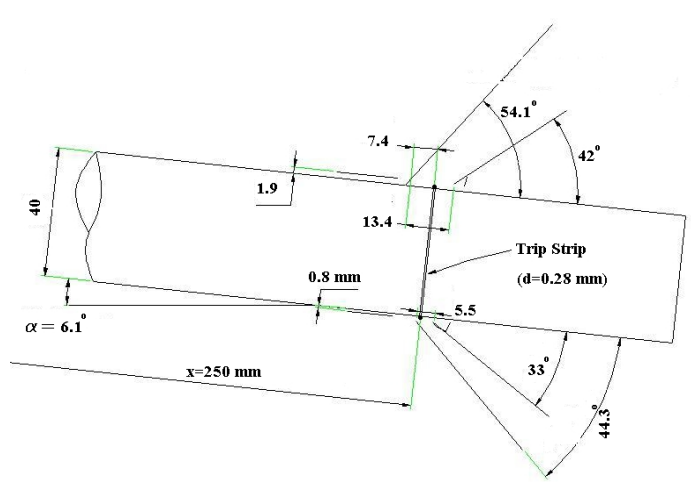

Shocks parameters

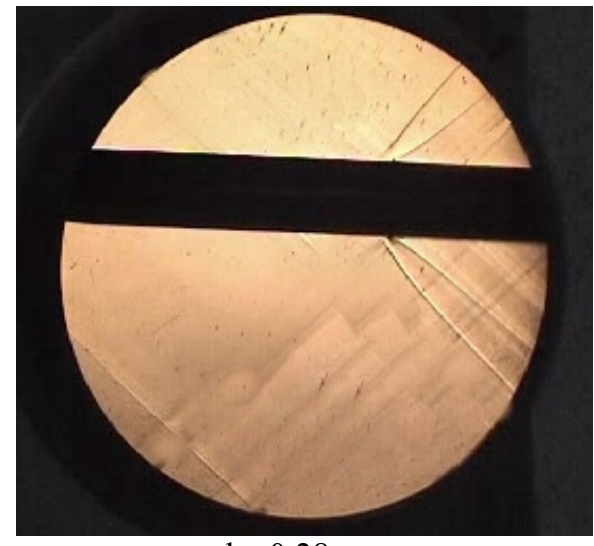

$\mathrm{d}_{\mathrm{ts}}=0.28 \mathrm{~mm}$

Fig. 7. Illustration of angles, distances and forms of the shocks relative to themselves and body, for the model 1 with $0.28 \mathrm{~mm}$ in diameter trip strip installed at $\mathrm{x} / \mathrm{d}=6.25$ and at $\alpha=6^{\circ}$.

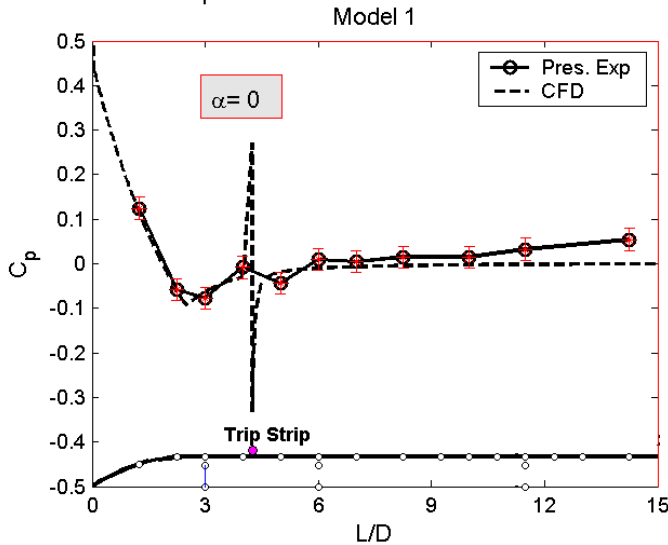

(a) Model $1, \mathrm{x}_{\mathrm{tr}} / \mathrm{d}=4.25$ and $\mathrm{d}_{\mathrm{tr}}=0.9 \mathrm{~mm}$.

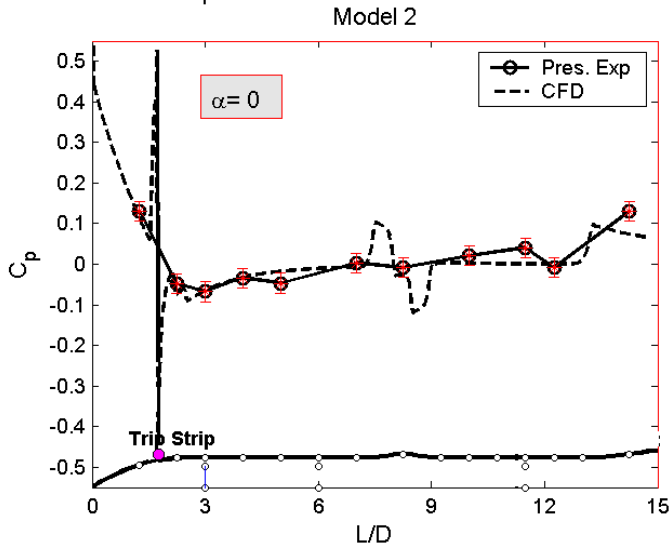

(b) Model 2, $\mathrm{x}_{\mathrm{tr}} / \mathrm{d}=1.75$ and $\mathrm{d}_{\mathrm{tr}}=1.14 \mathrm{~mm}$.

Fig. 8. Comparison of the experimental and the numerical longitudinal pressure distributions at $\alpha=0$. 
M.R. Heidari et al. / JAFM, Vol. 3, No. 2, pp. 53-63, 2010.

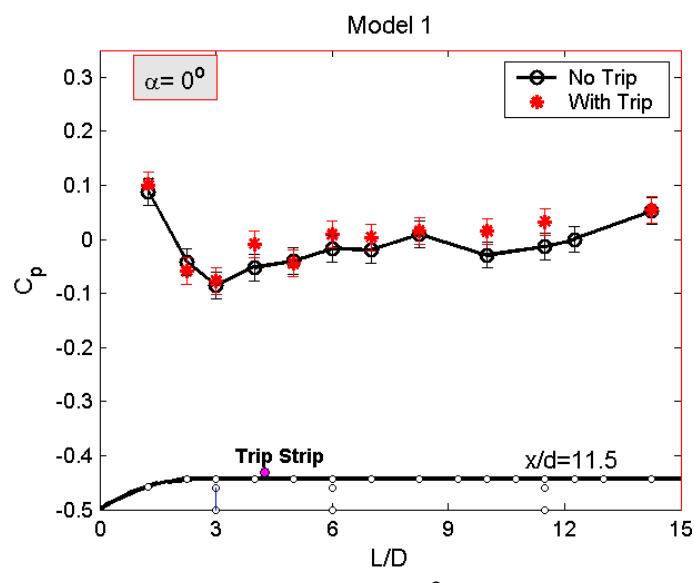

(a) $\alpha=0$

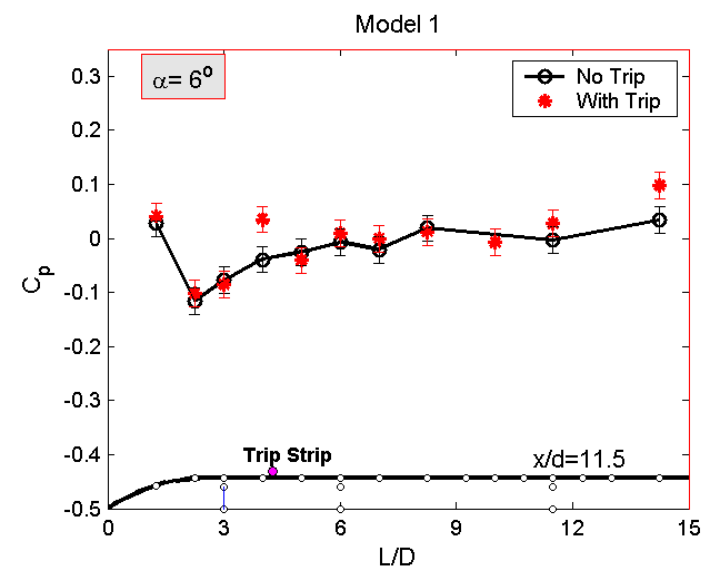

(b) $\alpha=6$

Fig. 9. Effect of boundary layer tripping on longitudinal pressure distributions for model 1 .

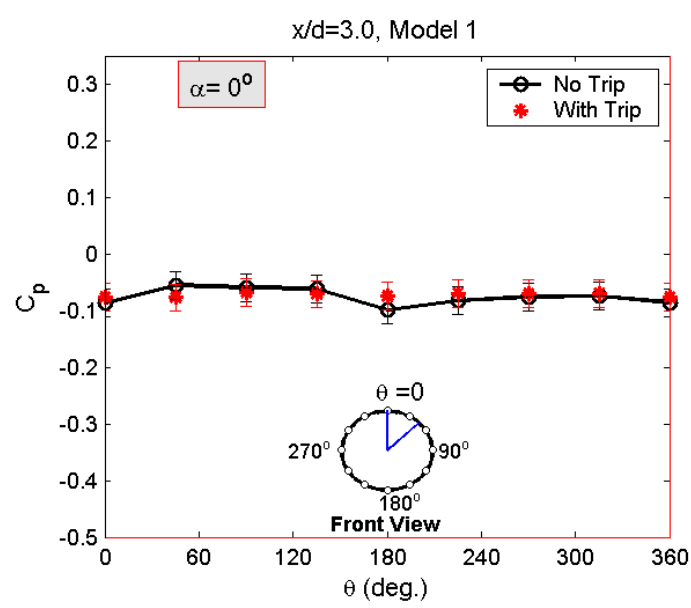

(a) $x / d=3$

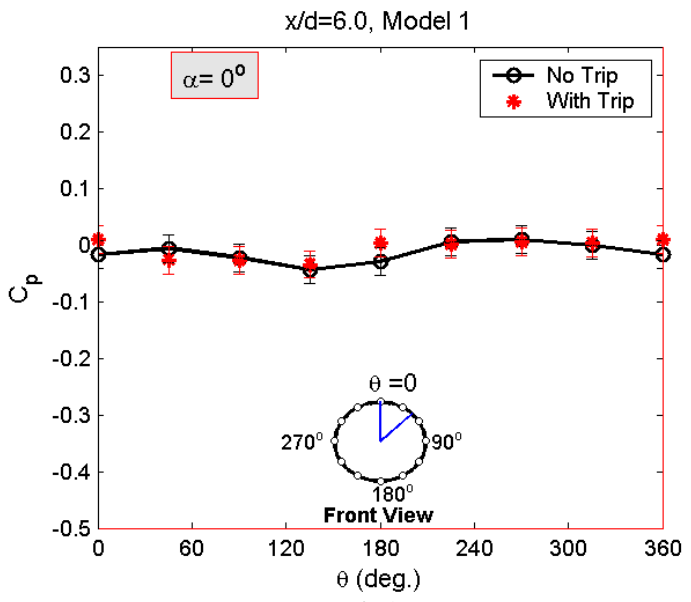

(b) $x / d=6$

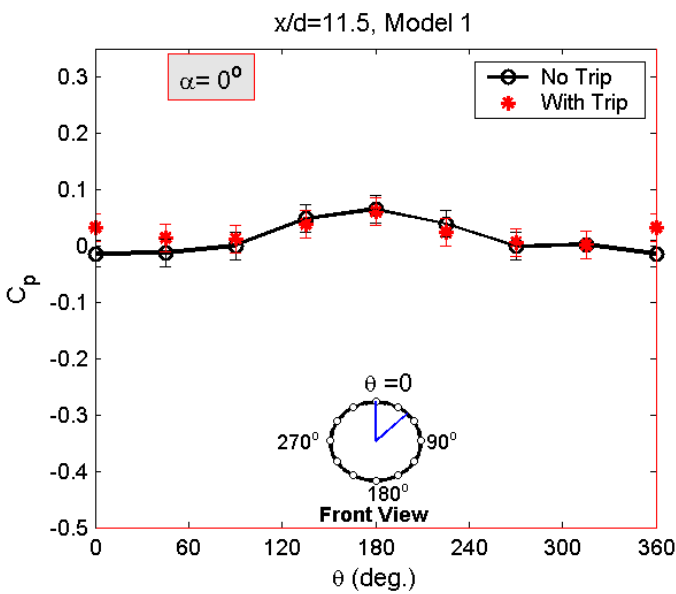

(c) $x / d=11.5$

Fig. 10. Effect of boundary layer tripping on the circumferential pressure distributions at $\alpha=0$ for model 1 . 


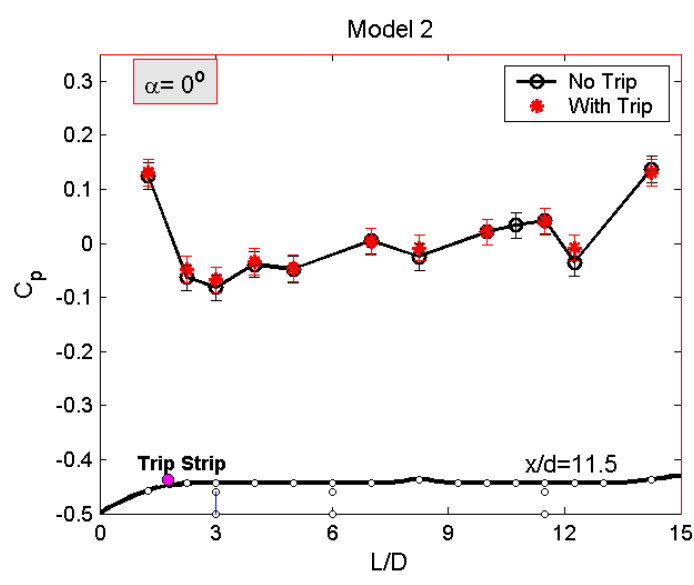

(a) $\alpha=0$

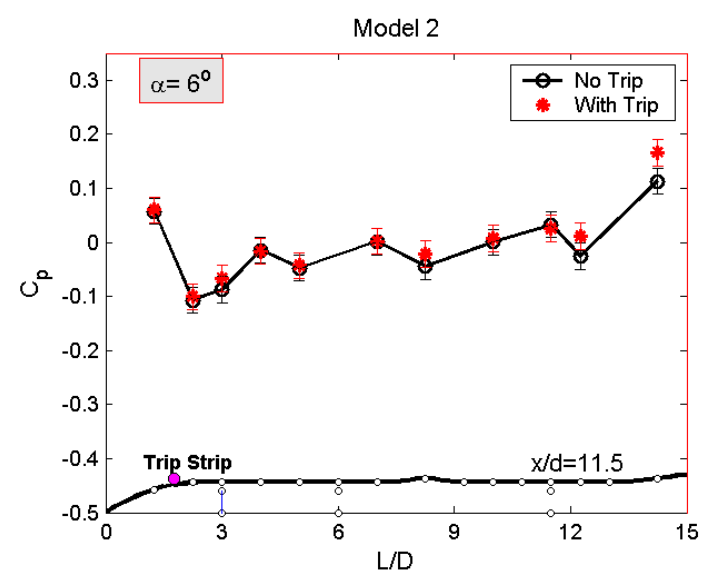

(b) $\alpha=6^{\circ}$

Fig. 4. Effect of boundary layer tripping on the longitudinal pressure distributions for model 2.

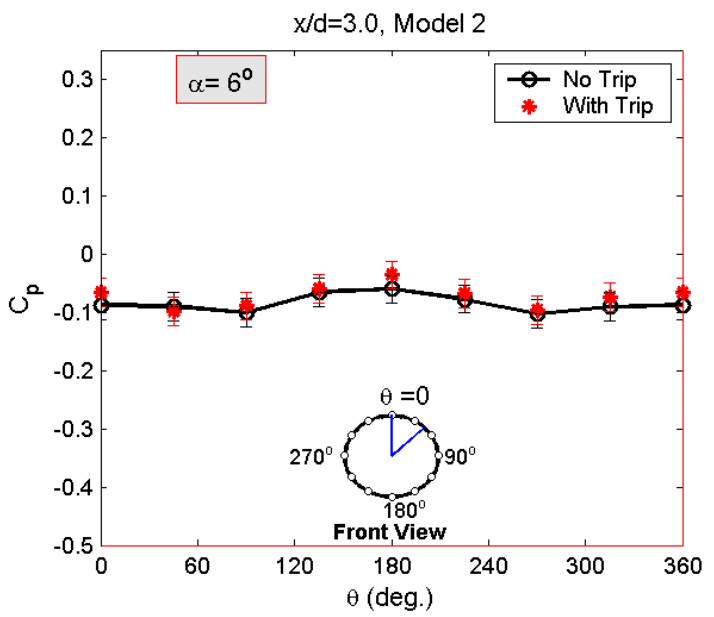

(a) $x / d=3$

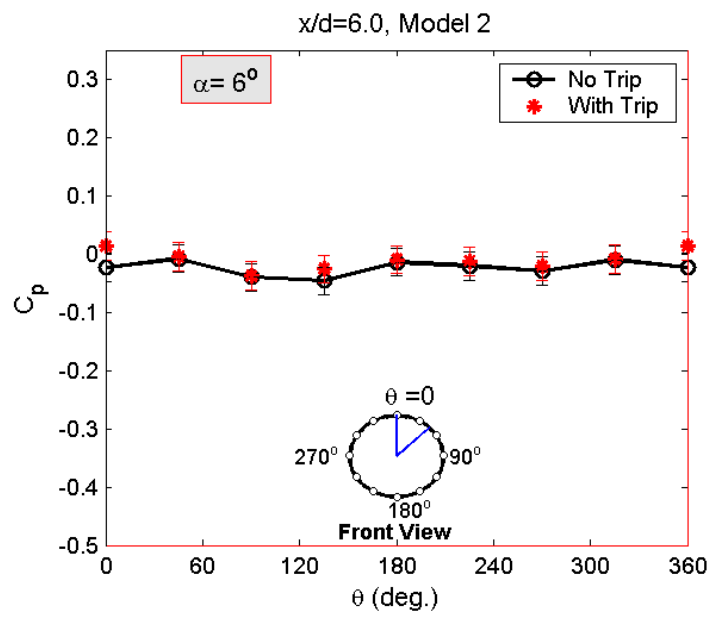

(b) $x / d=6$

Fig. 5. Effect of boundary layer tripping on the circumferential pressure distributions at $\alpha=6^{\circ}$ for model 2 .

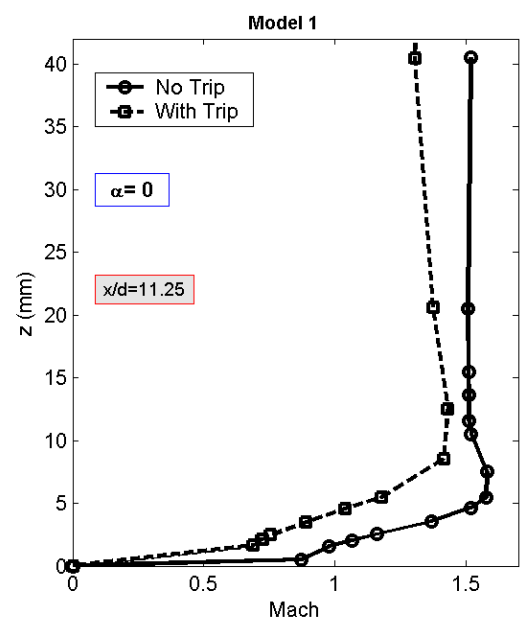

(a) $\alpha=0$

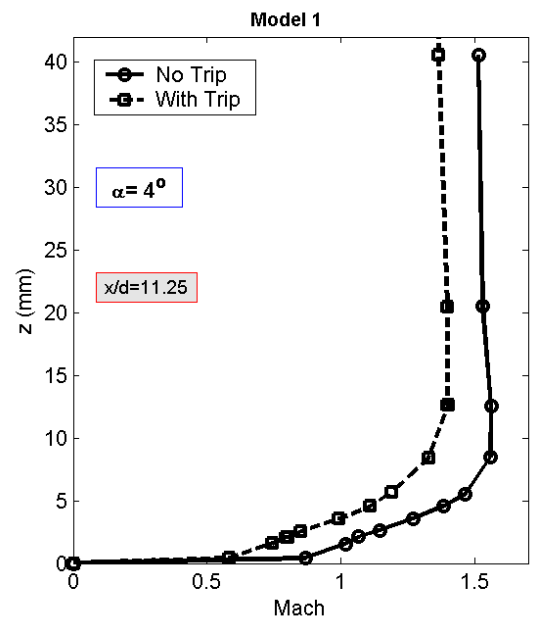

(b) $\alpha=4^{\circ}$

Fig. 6. Comparison of the boundary layer profiles for with/without trip strip on model 1 at $\mathrm{x} / \mathrm{d}=11.25$. 


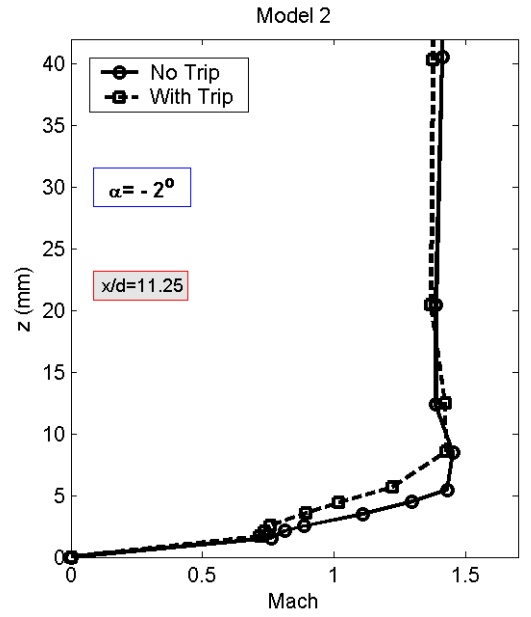

(a) $\alpha=-2^{\circ}$

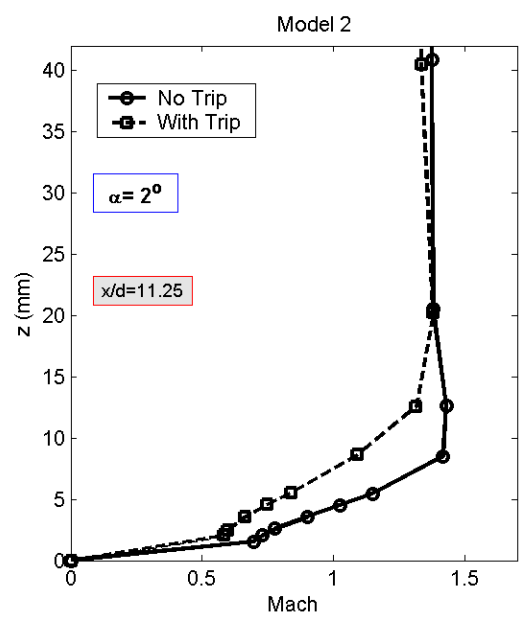

(c) $\alpha=2^{\circ}$

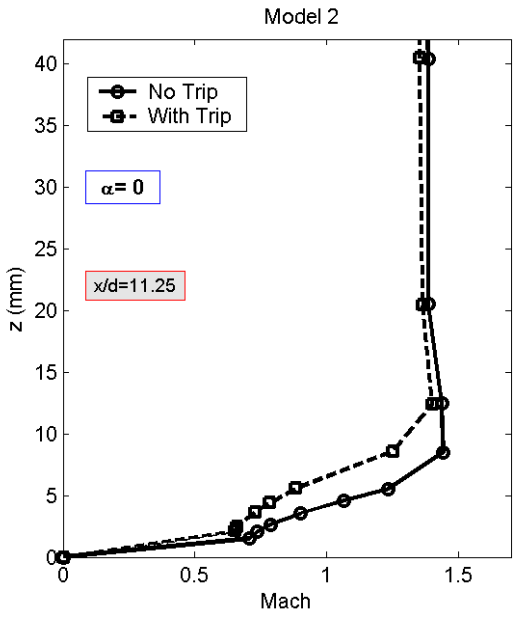

(b) $\alpha=0$

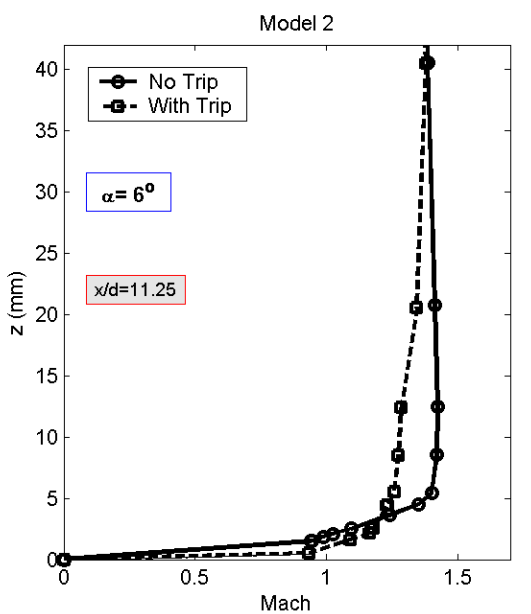

(d) $\alpha=6^{\circ}$

Fig. 7. Comparison of the boundary layer profiles for with/without trip strip on model 2 at $\mathrm{x} / \mathrm{d}=11.25$.

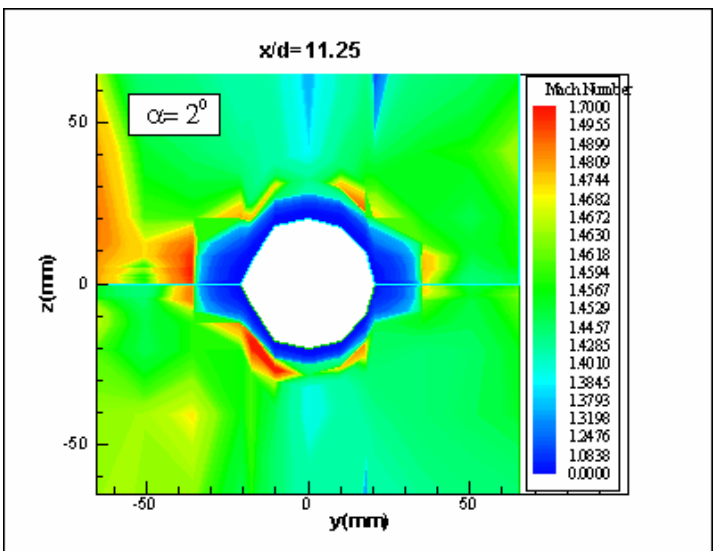

(a) Clean Model

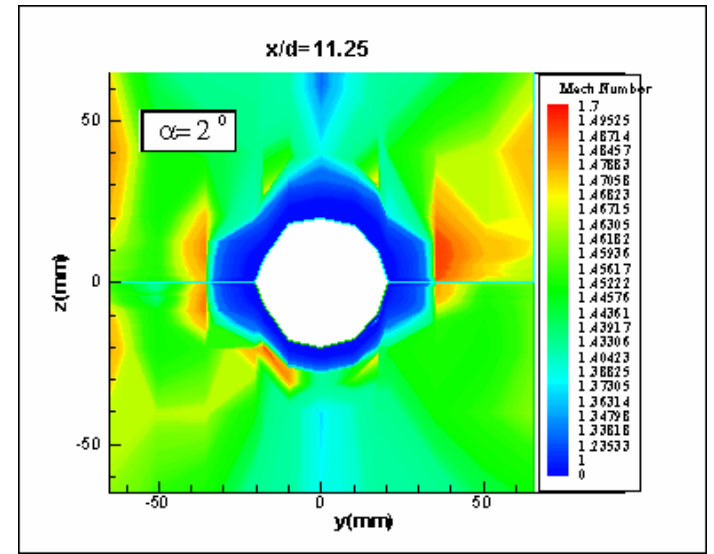

(b) Model with trip strip

Fig. 8. Effect of boundary layer tripping on Mach contours at $\mathrm{x} / \mathrm{d}=11.25$ and $\alpha=2^{\circ}$, for Model 2 . 